\title{
MD-SeeGH: a platform for integrative analysis of multi-dimensional
} genomic data

\author{
Bryan Chi*1, Ronald J deLeeuw ${ }^{1}$, Bradley P Coe ${ }^{1}$, Raymond T Ng1,2, \\ Calum MacAulay ${ }^{3}$ and Wan L Lam ${ }^{1}$
}

Address: ${ }^{1}$ Department of Cancer Genetics and Developmental Biology, British Columbia Cancer Research Centre, Vancouver, BC, Canada, ${ }^{2}$ Department of Computer Science, University of British Columbia, Vancouver, BC, Canada and ${ }^{3}$ Department of Cancer Imaging, British Columbia Cancer Research Centre, Vancouver, BC, Canada

Email: Bryan Chi* - bchi@bccrc.ca; Ronald J deLeeuw - rdeleeuw@bccrc.ca; Bradley P Coe - bcoe@bccrc.ca; Raymond T Ng - rng@cs.ubc.ca; Calum MacAulay - cmacaula@bccrc.ca; Wan L Lam - wanlam@bccrc.ca

* Corresponding author

Published: 20 May 2008

BMC Bioinformatics 2008, 9:243 doi:10.1/86/147/-2105-9-243
Received: 30 October 2007

Accepted: 20 May 2008

This article is available from: http://www.biomedcentral.com/I47I-2105/9/243

(c) 2008 Chi et al; licensee BioMed Central Ltd.

This is an Open Access article distributed under the terms of the Creative Commons Attribution License (http://creativecommons.org/licenses/by/2.0), which permits unrestricted use, distribution, and reproduction in any medium, provided the original work is properly cited.

\begin{abstract}
Background: Recent advances in global genomic profiling methodologies have enabled multidimensional characterization of biological systems. Complete analysis of these genomic profiles require an in depth look at parallel profiles of segmental DNA copy number status, DNA methylation state, single nucleotide polymorphisms, as well as gene expression profiles. Due to the differences in data types it is difficult to conduct parallel analysis of multiple datasets from diverse platforms.
\end{abstract}

Results: To address this issue, we have developed an integrative genomic analysis platform MDSeeGH, a software tool that allows users to rapidly and directly analyze genomic datasets spanning multiple genomic experiments. With MD-SeeGH, users have the flexibility to easily update datasets in accordance with new genomic builds, make a quality assessment of data using the filtering features, and identify genetic alterations within single or across multiple experiments. Multiple sample analysis in MD-SeeGH allows users to compare profiles from many experiments alongside tracks containing detailed localized gene information, microRNA, CPG islands, and copy number variations.

Conclusion: MD-SeeGH is a new platform for the integrative analysis of diverse microarray data, facilitating multiple profile analyses and group comparisons.

\section{Background}

Recent advances in global genomic profiling methodologies have enabled multi-dimensional characterization of biological systems. The deciphering of downstream effects of genetic and epigenetic alterations on expression patterns is paramount in understanding disease phenotype and requires the integration of segmental DNA copy number status, DNA methylation state and single nucle- otide polymorphism (SNP) status. The large scale generation of such data has created a need for robust software to integrate multiple large genetically linked data sets generated on diverse microarray platforms. Although several visualization software programs are available publicly (for example [1-5]), there is a growing demand for new bioinformatics tools that allow for the concerted analysis of multiple genome-wide experiments derived from differ- 
ent experimental platforms [6]. Blue Fuse [7] and CGH Analytics [8], two commercially available software tools, offer integrative analysis with expression data but neither contain the full feature set that we deem necessary (Table 1). SeeGH (v1.6) was initially developed to view primarily array CGH data [2] but as we continued to use and develop the software we realized that there was a need for the combined analysis of multi-platform data which required significant upgrades to the initial version of SeeGH. Here we present MultiDimensional-SeeGH (MDSeeGH) analysis platform, a powerful software tool that allows users to quickly and easily analyze genomic anchored datasets comprised from multiple genomic experiments (Figure 1).

\section{Implementation}

MD-SeeGH was created using Borland's C++ Builder6 development platform. MySQL is used as the backend database server which is freely available for download. The MD-SeeGH software was developed and tested on Windows XP and Vista. The software and documentation are publicly available online [9].

From our interaction with researchers and clinicians, we note that some of the key features required by integrative analysis software for handling diverse genomic datasets are: (1) flexibility (2) data quality assessment, (3) visualization (4) single and multiple sample analyses, (5) multi group analyses, and (6) comprehensive reporting. To highlight how MD-SeeGH performs these functions, we discuss the parallel analysis of genomic and epigenomic array comparative genomic hybridization (CGH) data as well as the analysis of multidimensional data sets includ- ing gene expression, comparative genomic hybridization, differential methylation, and single nucleotide polymorphisms.

\section{Results and discussion}

The following sections describe the flow chart summarizing the functional modules of MD-SeeGH (Figure 1).

\section{Data tracking, preprocessing and import of data}

Microarray data captured after hybridization, scanning, and spot finding are imported into MD-SeeGH as tabdelimited text files. At this time each dataset can be annotated to facilitate data tracking, easy recall, and group definition. Clinical information can also be entered and associated with each dataset. Microarray image data are commonly normalized to remove intensity and spatial biases. For example, the output of a stepwise normalization algorithm, CGH-Norm, is seamlessly imported into MD-SeeGH [10].

\section{Flexible genome mapping and annotation}

To relate array spot information to a specific genomic map location, it is important to use the appropriate genome build (e.g. UCSC Human Genome Freeze Mar 2006/ hg18). We have embedded the genomic locations of array features within MD-SeeGH for all available genome builds utilized by the common genomic microarray platforms. New mapping information (future builds) can be easily imported into MD-SeeGH using tab-delimited text files containing base pair information for each array feature. This provides the user with the ultimate flexibility of remapping entire datasets against any genomic build without having to manually transform each individual

Table I: Feature comparison of integrative analysis platforms

\begin{tabular}{|c|c|c|c|c|c|c|c|c|c|c|}
\hline & MD-SeeGH & VAMP/CAPweb & ISACGH & CGH Analytics & CGHPRO & CGH Explorer & Blue Fuse & ArrayCyGHt & $\mathrm{M}-\mathrm{CGH}$ & SeeGHvl.6 \\
\hline \multicolumn{11}{|c|}{ Segmentation } \\
\hline \multicolumn{11}{|c|}{ Normalization } \\
\hline \multicolumn{11}{|c|}{$\begin{array}{l}\text { Integrative Analysis with } \\
\text { Expression data }\end{array}$} \\
\hline \multicolumn{11}{|c|}{ Multiple Sample Visualization } \\
\hline \multicolumn{11}{|c|}{ Gene Tracks } \\
\hline \multicolumn{11}{|c|}{$\begin{array}{l}\text { Links to external websites } \\
\text { (ie. NCBI, UCSC, etc.) }\end{array}$} \\
\hline \multicolumn{11}{|c|}{$\begin{array}{l}\text { Mapping files (different } \\
\text { Genomic Builds) }\end{array}$} \\
\hline \multicolumn{11}{|c|}{$\begin{array}{l}\text { Integration of third party } \\
\text { analysis tools }\end{array}$} \\
\hline \multicolumn{11}{|c|}{ Frequency Plot } \\
\hline \multicolumn{11}{|c|}{ Heatmap } \\
\hline References & & {$[6,20]$} & [2I] & [8] & [I] & [3] & [7] & [22] & [5] & [2] \\
\hline
\end{tabular}




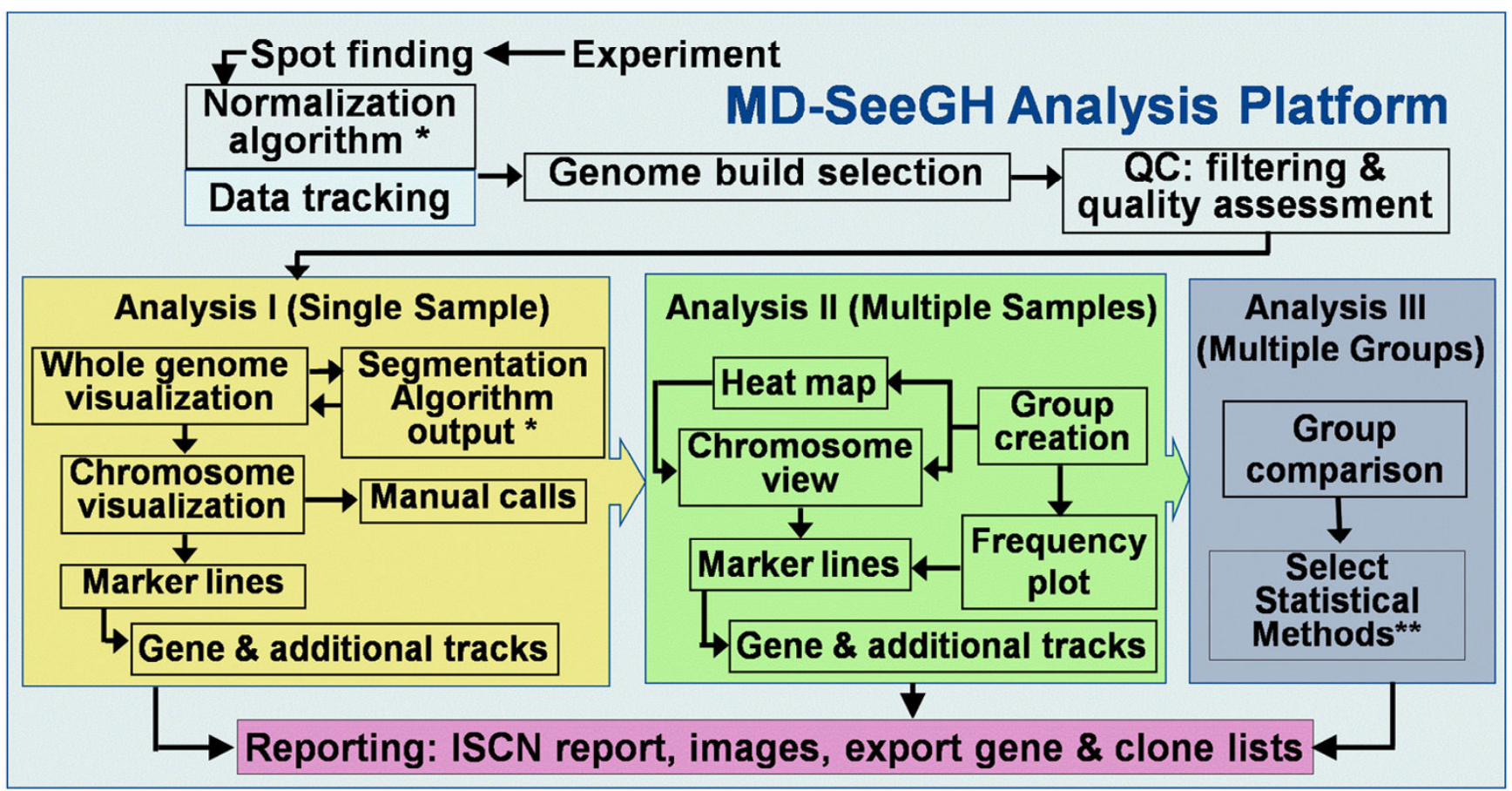

* MD-SeeGH Analysis Platform includes normalization software CGH Norm and segmentation software CNA HMMer.

** Statistical analysis must be done externally.

Figure I

Flow Diagram Summarizing the MD-SeeGH Platform.

dataset and reload them into MD-SeeGH [see Additional file 1].

\section{Quality assessment of data}

MD-SeeGH allows filtering of spot data based on standard deviation of replicate measurements and spot signal to noise ratios based upon user-defined parameters. The percentage of spots discarded by filtering and the average signal to noise ratio are displayed for each experimental dataset. A recently described phenomenon in array CGH experimentation (regardless of array platform used) has been the identification of a recurrent artefact pattern that is independent of the copy number status $[11,12]$. We have created a tool to measure and compensate for this identified recurrent baseline pattern (noise) within array CGH experiments [11] [see Additional file 1].

\section{Detection of genetic alterations}

Once the imported data has undergone the appropriate quality assessment, analysis can begin with the identification of alterations for each sample (Analysis I - Figure 1). Many segmentation algorithms have been developed to identify regions of alteration, each with their unique strengths/weaknesses[13]. Given that each microarray platform may require a specific segmentation algorithm, for example a modified Hidden Markov Model for segmentation of array CGH data [14]. MD-SeeGH allows the user to import the output from such algorithms as CNA HMMer, DNAcopy and aCGH Smooth [14-16]. (Can also import any dataset where each spot is annotated with a call). The result of each segmentation output is displayed beside each measured experimental data feature to assist the user in assigning copy number representation to the data within an experiment (Figure 2) [see Additional file $1]$.

\section{Gene and additional tracks}

Accessible information embedded in MD-SeeGH includes all annotated RefSeq genes, microRNAs[17], CpG islands[18], and natural copy number variations (CNVs) [19]. However, we provide the user with the flexibility to display any genomic annotated fields as a track beside the experimental data (Figure 3a). Data within gene tracks can be selected to display information about each gene from multiple sources (OMIM, Entrez mRNA, Entrez protein, Pubmed, and UCSC genome browser). The gene and additional tracks allow the user to determine if a specific spot on their array overlaps with a specific gene, microRNA, CNV, etc. Up to 4 tracks are visible at all zoom levels during analysis and visualization. Alternately, an entire 


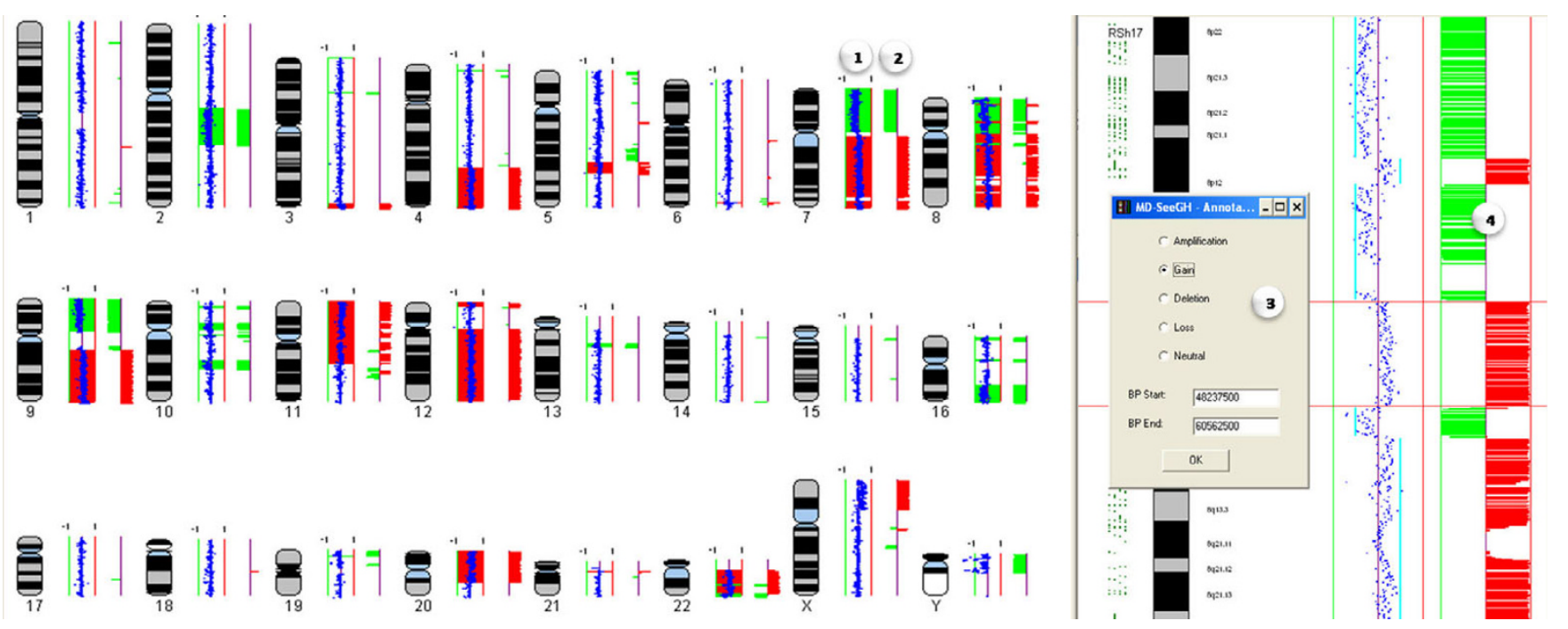

Figure 2

Identification and Annotation of Altered Regions. The Annotate Regions option is an analysis tool that allows you to record regions of interest (i.e. amplifications, deletions), save them to the database, and create ISCN reports. Annotating regions can be used side by side with segmentation probabilities to verify the called regions and can also be used to compare amplification and deletions across multiple samples or create Frequency Plots. Numbers indicate genomic view of (I) annotated regions and (2) segmentation calls, and chromosome view of (3) annotation form where user can mark the region as an amplification, gain, deletion, loss or neutral region and (4) segmentation calls which aid in making the calls.

region can be displayed via the UCSC genome browser at the touch of a button [see Additional file 1].

\section{Multiple sample analyses}

In MD-SeeGH, data from up to 50 experiments can be aligned for direct comparison allowing for cross platform analysis or viewing multiple patient samples from the same disease type (Analysis II - Figure 1) (Figure 3). Multiple samples are viewed one chromosome at a time and can be easily changed via a chromosome drop-down box. There is no limit on the density of arrays when viewing multiple samples. Of course the larger the arrays the longer it takes to load. During testing of the Multiple Alignment feature we were able to load 50 Agilent $244 \mathrm{k}$ arrays in 1 minute and 20 seconds on a computer with 2 $\mathrm{GB}$ of RAM and a $2.7 \mathrm{Ghz}$ processor. On the same machine, 50 SMRT $32 \mathrm{~K}$ arrays took less than 15 seconds to load. In addition, up to 100 experiments can be analyzed and summarized as a heatmap (Figure 4). The heatmap is generated by calculating a moving average across each experiment and allows for a quick way to find regions of interest across a large number of experiments. A given region of interest identified on the heatmap can be further investigated in detail by directly switching to multiple alignment of individual profiles. MD-SeeGH also has the ability to analyze up to 1000 samples as a frequency plot showing percent of samples altered (Figure 5 ). The frequency plot feature gives the user the ability to identify minimal regions of interest across large datasets.
Frequency plots can be created within MD-SeeGH for any datasets from the same array platform that have called data. The frequency of alteration is calculated for each spot of the selected array platform. When creating frequency plots within MD-SeeGH, the maximum density allowed is 25,000 spots per chromosome for a total of 600 $\mathrm{K}$ spots in the array. Frequency plot data can also be created externally and loaded into MD-SeeGH. When analysing frequency plots, whole genome and individual chromosome plots are available to the user [see Additional file 1].

\section{Platform independence and integrative analyses of multi- dimensional datasets}

Any data that is tied to a genomic base pair position can be loaded into MD-SeeGH. This includes single channel Affymetrix SNP arrays and double channel Agilent, Nimblegen, Illumina, and SMRT arrays. Within multiple sample analyses, it is not a requirement that all data be created from the same microarray platform. This capability can be utilized to assess the differing characteristics of microarray platforms (Figure 3 ) or combine data derived from the latest platforms with data created using older platforms. This functionality is increasingly desirable to analyze multidimensional datasets, for example, the integration of methylation patterns, copy number alterations and single nucleotide polymorphisms (Figure 6a,b). However, its main advantage is in analyzing gene expression changes in the context of these genetic features (Figure 6c). 


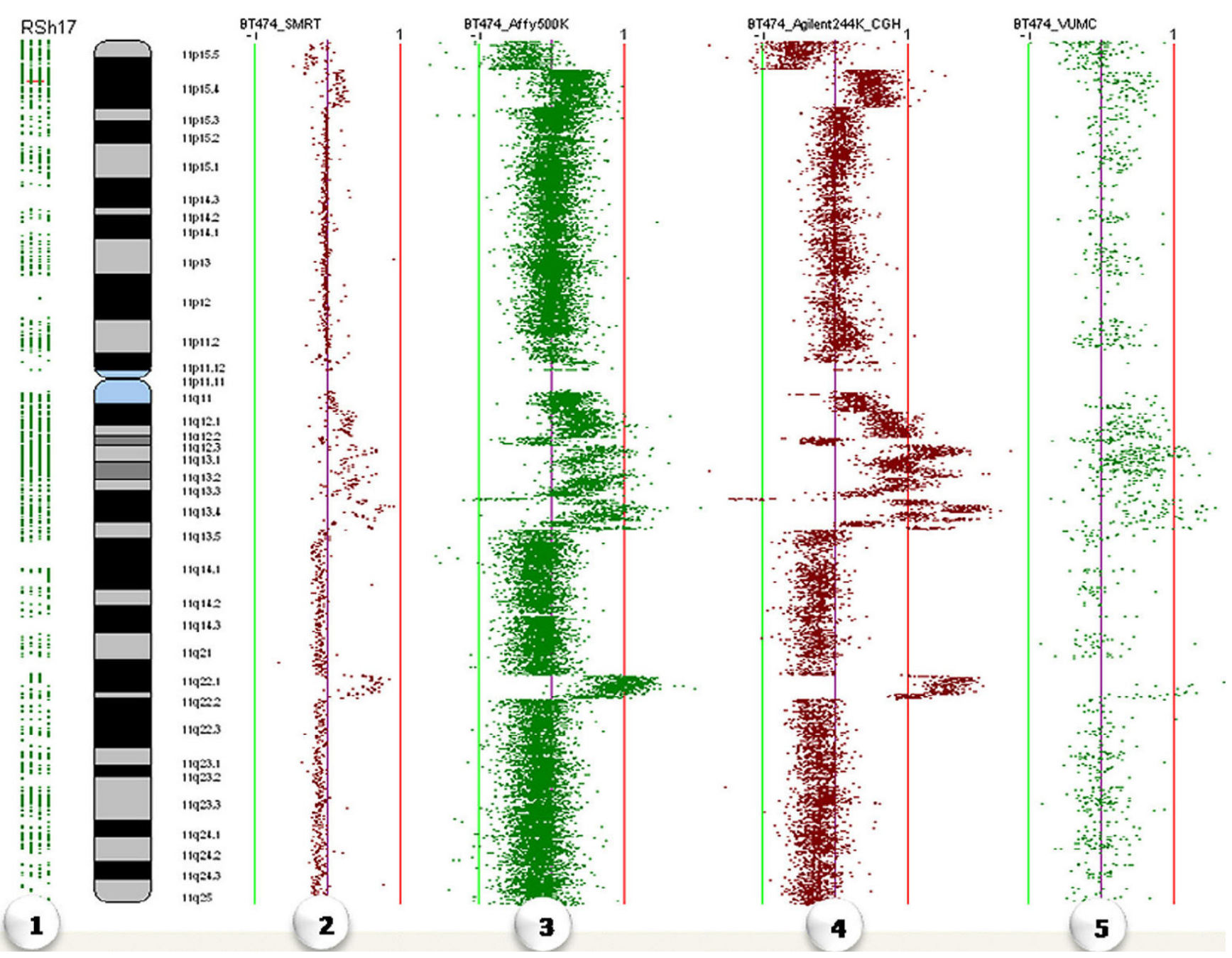

Figure 3

Multiple Analyses of Different Platforms. Comparison of the same sample (BT474 cell line) across the following different array platforms: (I) RefSeq GeneTrack, (2) SMRT array, (3) Affymetrix GeneChip human mapping $500 \mathrm{~K}$ set, (4) Agilent 244A, and (5) VUMC MACF human $30 \mathrm{~K}$.

\section{Comparative analysis of multiple groups}

An additional level of analysis is the ability to compare two groups of experiments to identify differences between them. In MD-SeeGH this is achieved through the comparison of frequency plots (Figure 7). Permutation testing, Fisher's exact tests, and other statistical tests can be easily conducted using data exported from MD-SeeGH. These statistical analyses provide p-values for the differences between the two groups [see Additional file 1].

\section{Exporting results and analysis reporting}

MD-SeeGH provides three main ways to export data. Firstly, any image can be saved as either a jpeg or a bitmap file. Secondly, noting that new analysis algorithms are constantly being developed, we built in the ability to export data from MD-SeeGH in a tab-delimited text format that can be readily manipulated with other software/ statistical packages. Finally, in a clinical or repetitive standardized analysis setting, the attachment of an entire array CGH data file to a report is unrealistic; therefore, we allow direct generation of a cytogenetic report, formatted with the latest ISCN standard for array experiments (Figure 8) [see Additional file 1].

\section{Conclusion}

In conclusion, we have developed a new platform for the integrative analysis of diverse microarray data, facilitating multiple profile analyses and group comparisons. 


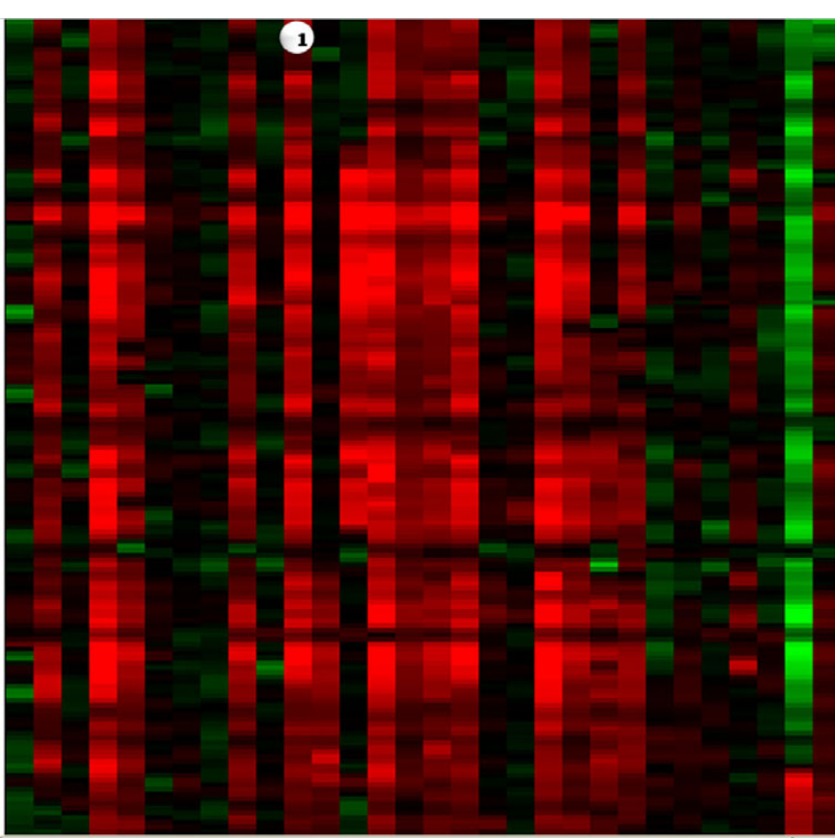

Figure 4

Heatmap for Defining Recurring Features. Heatmap allows the user to analyze up to 100 samples and find common regions of amplification or deletion across groups of samples. (I) Each column represents a moving average heatmap of a single sample. Amplifications are shaded red and deletions are shaded green. The greater the moving average ratio the brighter the red $(-)$ or green $(+)$.

\section{Availability and requirements \\ Project name: MD-SeeGH}

Project home page:http://www.arraycgh.ca

Operating system: Microsoft Windows XP, Microsoft Windows Vista

Programming language: $\mathrm{C}++, \mathrm{SQL}$

Other requirements: MySQL database

License: Academic software license must be agreed upon during installation.

Any restrictions to use by non-academics: Yes

\section{Authors' contributions}

$\mathrm{BC}$ was the principle programmer of the source code. RJdL, BPC and RTN contributed ideas for software features and requirements. CM and WLL are principle investigators of this work. All authors contributed to writing the manuscript. All authors read and approved the final manuscript.
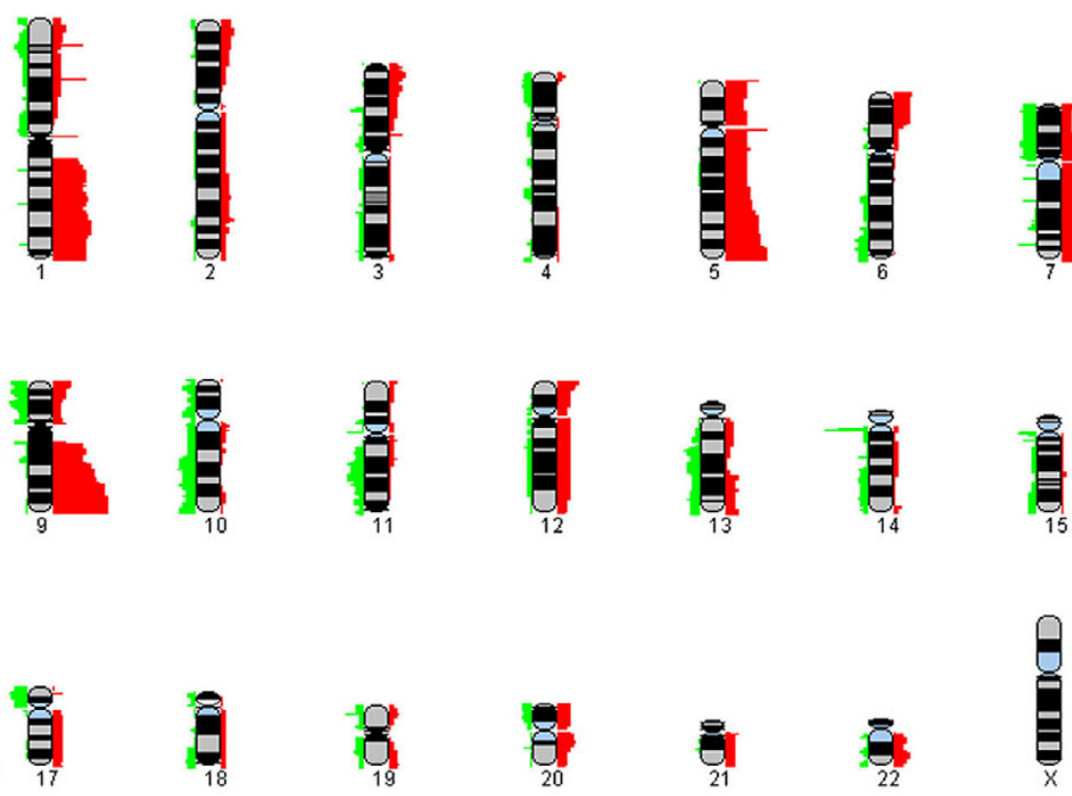
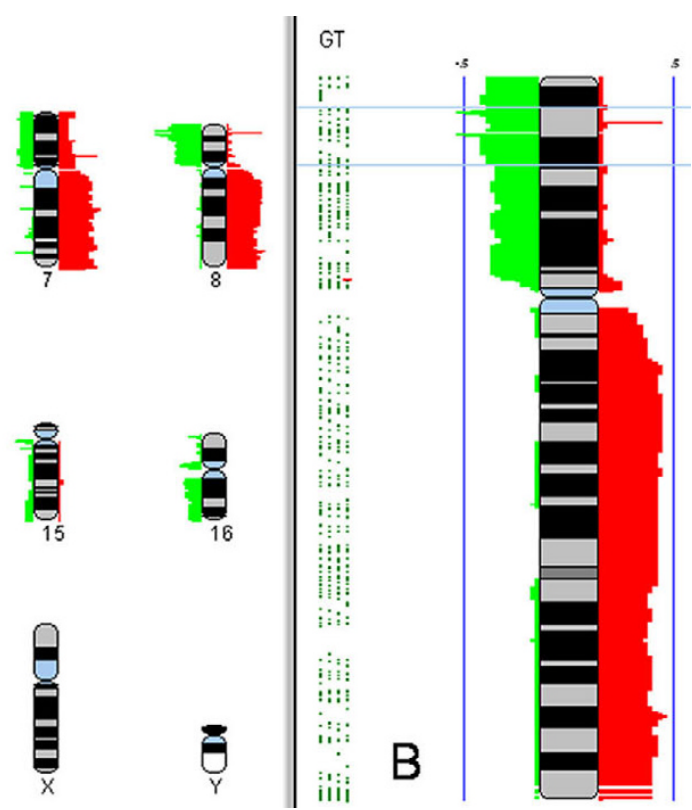

\section{Figure 5}

Frequency Plot. The Frequency Plot can be used to analyze a group of samples and find minimal regions of amplifications or deletions. Frequency plot scoring for up to 1000 samples can be created within SeeGH or created externally and loaded into SeeGH. Once loaded each sample is stored in the SeeGH database. Amplifications are shaded red and deletions are shaded green. Left panel (A) shows genome view and right panel (B) shows the chromosome view. 


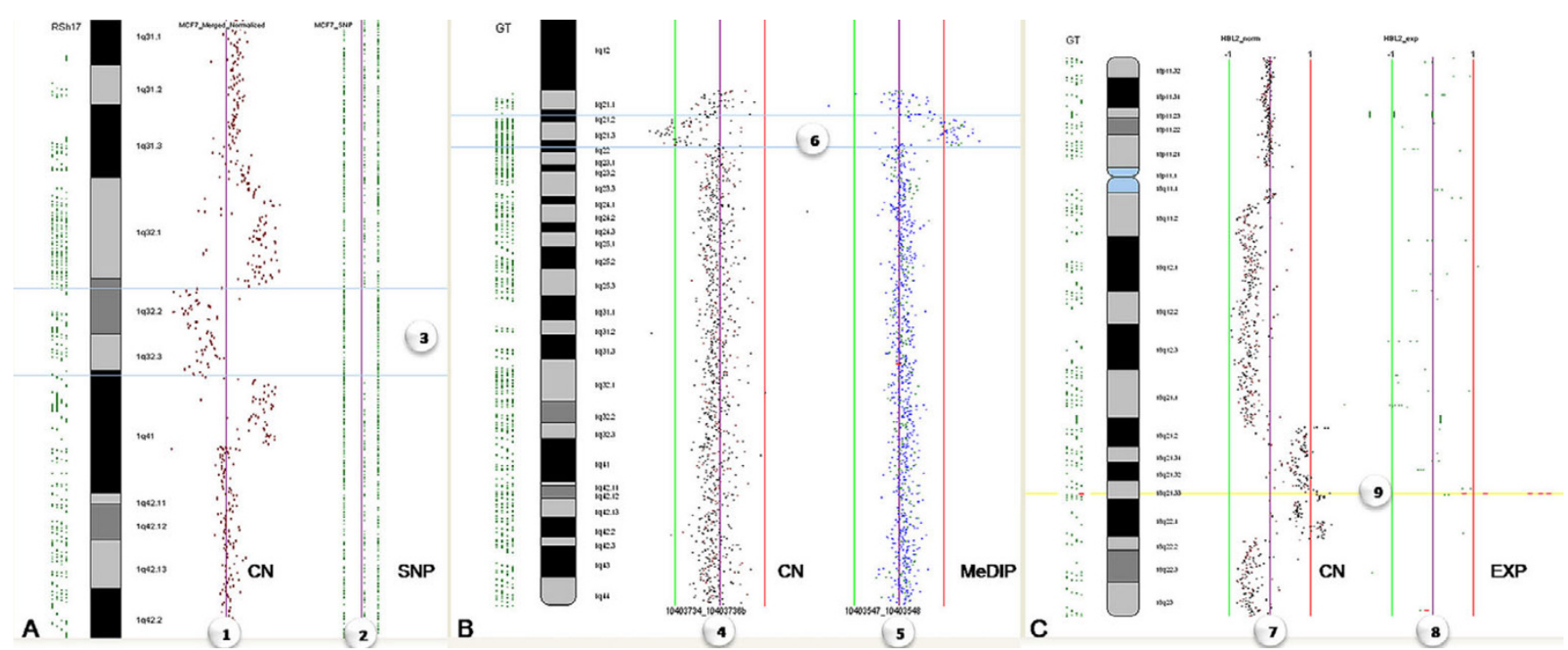

Figure 6

Analysis of Multi-Dimensional Data: Integration of Different Types of Data. a. Integration of copy number (CN) data in the context of SNP profile for MCF7 cells. (I) SMRT array CGH profile displayed alongside (2) Affymetrix SNP array-Homozygous AA on left, Heterozygous AB in middle, Homozygous BB on right. Region between the blue lines (3) shows a copy number loss (left) on chromosome I associated with LOH (right). b. Integration of epigenetic and genomic profiles. (4) Methylated DNA immunoprecipitation (MeDIP) array CGH profile displayed alongside a (5) SMRT array CGH. Region between the blue lines (6) shows both hypomethylation (left) and copy number change (right). c. Integration of Array CGH and Lymphochip cDNA Gene Expression. (7) SMRT array CGH profile displayed alongside a (8) cDNA expression (EXP) profile (Lymphochip). Yellow highlighted region (9) shows a BCL2 gain and overexpression.
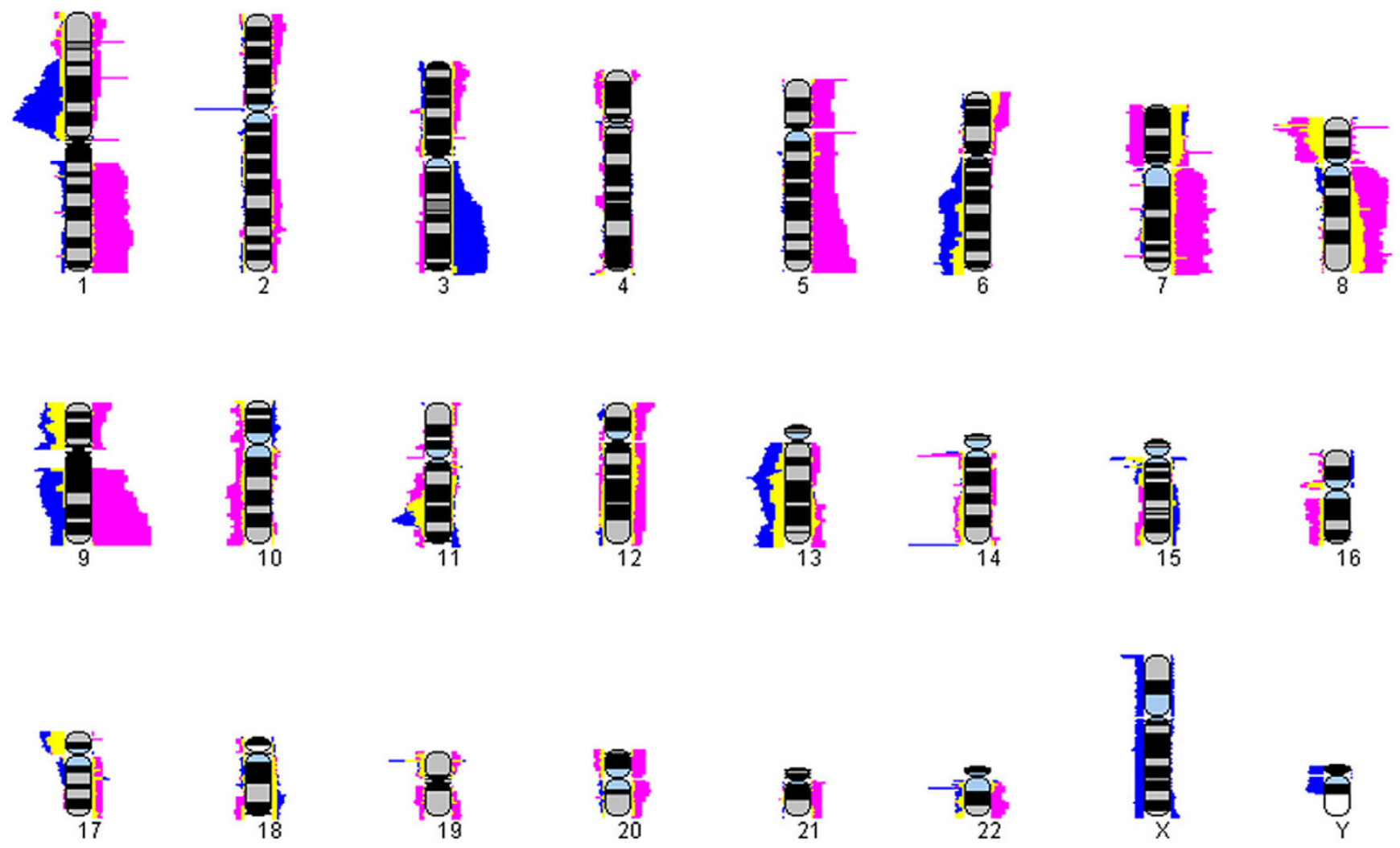

Figure 7

Group Comparison. Frequency plot comparison of two different groups (derived from two frequency plot datasets) representing different disease types. Once frequency plots have been loaded/created in SeeGH the user can compare two frequency plots using the overlay feature. Each group is a different color (Group 1 - Fuschia, Group 2 Blue) and any overlapping regions are a third color (Intersection - Yellow). This is a useful feature to determine similarities and differences between two groups of samples. 


\section{$(1)$}
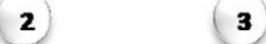

arx cgh 2q13q24.2(N0726P14->N0099D08) x1

arr cgh 3p26.3 (N0359E09->N0203I11) x1

arr cgh 3p21.31 (N0427P05->N0095N04) x1

arr cgh 3q28q29 (N0739N07->N0496H01) x3

arr cgh 4p16.1 (M2205P10->M2310D22) x1

arr cgh 4q28.3q35.2 (N0764 F10->N0652 J12) x3

arr cgh 5q23.1q31.1 (M2028 K10->N0622006) x3

arr cgh 6q26 (N0159M09->M2034B02) x3

arr cgh 7p11.1 (M2245C05->N0548K12) x1

arr cgh 7q11.21q35 (F0650L09->N0165B05) x3

arr cgh $7 \mathrm{q} 36.1 \mathrm{q} 36.3$ (N0478 $\mathrm{H} 20->\mathrm{N} 0133 \mathrm{~J} 16) \times 3$

arr cgh $8 \mathrm{p} 23.2$ (N0130K11->N0546K23) x1

arr cgh $8 \mathrm{p} 23.2$ (N0357H01->N0798G03) 1

arr cgh 8p23.2p23.1 (M2015I08->N0143J23) x1

arr cgh 8p23.1 (M2339A14->N0593D05) x3

arr cgh 8p23.1p12 (N0437P14->N0139G09) x1ㅣ

arx cgh 8p12(N0722M19->N0597D18) x3

arr cgh 8p12q11.21 (N0313F12->N0769I06) x1

arr cgh 8q11.21q12.1 (N1130I03->N0032A10) x3

arr cgh 8q12.1q12.3 (N0092N03->M2120K11) $\times 1$

arr cgh 8q12.3q22.3 (N0422P09->N0318M02) x3

arr cgh 8q23.1 (M2010G21->N0042I05) x3

arr cgh 8q23.3q24.13 (N0608K12->N0324I17) x3

arr cgh 8q24.13q24.3 (N0065D17->M2192A17) x3

arr cgh 9p11.2(M2260A14->N0603K04) x1

arr cgh 9q13q34.3 (N0088I18->N0424E07) x3

arr cgh 10p14 (N0045N19->N0306J07) x1

arr cgh 10p11.23p11.1 (N0427M08->F0547M11) x1

arr cgh 10q11.22(N0343H22->N0192A16) x1

arr cgh 10q22.3q23.31 (N0407P23->N0341B24) x1

arx cgh 11p15.5q14.1 (N0182E22->N0596I05) x3

arr cgh 12p13.31(F0492B24->N0277J24) x3

arr cgh 12q11q24.33 (N0159F08->N0386I08) $\times 3$

arr cgh 13q13.3q14.11 (N0804K04->N0140K22) x1

arr cgh 16p13.3p13.2 (N0613G15->N1372C07) x1

arr cgh 16p11.2p11.1 (N0170L03->N0295H06) x1

arr cgh 16q22.1q24.3 (N0421NO4->NO533D19) x1

arr cgh 19p13.2 (N0743010->N0203K06) x1

arr cgh 20p13q13.12(D2506E02->N0770M01) x3

arr cgh 21q11.2 (N0459P06->N0129M19) x3

arx cgh 22q11.1q12.3 (M2177M20->N0806I19) x3

arr cgh 22q12.3 (N0452N11->N0108P21) x1

arr cgh 22q12.3q13.31 (N0768B09->N0435J19) x3

arr cgh 22q13.31q13.33(N0620R14->M2039k23) x1

\section{Figure 8}

Sample ISCN report exported from MD-SeeGH. Tabdelimited ISCN report exported from MD-SeeGH. Regions are noted with (I) chromosome banding position, (2) first and last clone/feature of the region, and (3) whether it is an amplification or deletion. Amplifications or copy number gain is marked as ' $x 3$ ', while deletions or copy number loss is marked as ' $\mathrm{xl}$ '.

\section{Additional material}

Additional file 1

Supplementary figures

Click here for file

[http://www.biomedcentral.com/content/supplementary/1471-

2105-9-243-S1.pdf]

\section{Acknowledgements}

We thank Spencer Watson and Dr. Doug Horsman for useful discussion and Andre Soesilo, Tony Qin, and Philip Wang for help with software development. This work was supported by funds from Genome Canada/British Columbia, Canadian Institutes for Health Research, Canadian Breast Cancer Research Alliance and NIH (NIDCR) ROI DEI 5965.

\section{References}

I. Chen W, Erdogan F, Ropers HH, Lenzner S, Ullmann R: CGHPRO - a comprehensive data analysis tool for array CGH. BMC Bioinformatics 2005, 6:85.

2. Chi B, DeLeeuw RJ, Coe BP, MacAulay C, Lam WL: SeeGH--a software tool for visualization of whole genome array comparative genomic hybridization data. BMC Bioinformatics 2004, 5: I3.

3. Lingjaerde OC, Baumbusch LO, Liestol K, Glad IK, Borresen-Dale AL: CGH-Explorer: a program for analysis of array-CGH data. Bioinformatics 2005, 2 I(6):821-822.

4. Lockwood WW, Chari R, Chi B, Lam WL: Recent advances in array comparative genomic hybridization technologies and their applications in human genetics. Eur J Hum Genet 2006, I 4(2): | 39- 148.

5. Wang J, Meza-Zepeda LA, Kresse SH, Myklebost O: M-CGH: analysing microarray-based CGH experiments. BMC Bioinformatics 2004, 5:74.

6. La Rosa P, Viara E, Hupe P, Pierron G, Liva S, Neuvial P, Brito I, Lair S, Servant N, Robine N, Manie E, Brennetot C, Janoueix-Lerosey I, Raynal V, Gruel N, Rouveirol C, Stransky N, Stern MH, Delattre O, Aurias A, Radvanyi F, Barillot E: VAMP: visualization and analysis of array-CGH, transcriptome and other molecular profiles. Bioinformatics 2006, 22(I 7):2066-2073.

7. Blue Fuse Software [http://www.cambridgebluegnome.com/blue fuse.htm]

8. CGH Analytics Software [http://www.chem.agilent.com/Scripts/ PDS.asp?!Page=29457]

9. Flintbox [http://www.flintbox.com/technology.asp?page=706]

10. Khojasteh M, Lam WL, Ward RK, MacAulay C: A stepwise framework for the normalization of array CGH data. BMC Bioinformatics 2005, 6:274.

II. Blesa D, Suela J, Melchor L, Alvarez S, Largo C, Ferreira B, Calasanz MJ, Cifuentes F, Cigudosa JC: Artefacts in aCGH: La Grande Motte, France. ; 2006.

12. Marioni JC, Thorne NP, Valsesia A, Fitzgerald T, Redon R, Feigler H, Andrews TD, Stranger BE, Lynch AG, Dermitzakis ET, Carter NP, Tavare S, Hurles ME: Breaking the waves: improved detection of copy number variation from microarray-based comparative genomic hybridization. Genome Biology 2007, 8(R228):.

13. Lai WR, Johnson MD, Khucherlapati R, Park PJ: Comparative analysis of algorithms for identifying amplifications and deletions in array CGH data. Bioinformatics 2005, 2 I (19):3763-3770.

14. Shah SP, Xuan X, DeLeeuw RJ, Khojasteh M, Lam WL, Ng R, Murphy $\mathrm{KP}$ : Integrating copy number polymorphisms into array $\mathrm{CGH}$ analysis using a robust HMM. Bioinformatics 2006, 22( I4):e43 I-9.

15. Jong K, Marchiori E, Meijer G, Vaart AV, Ylstra B: Breakpoint identification and smoothing of array comparative genomic hybridization data. Bioinformatics 2004, 20( I 8):3636-3637.

16. Venkatraman ES, Olshen AB: A faster circular binary segmentation algorithm for the analysis of array CGH data. Bioinformatics 2007, 23(6):657-663. 
17. Griffiths-Jones S, Grocock RJ, van Dongen S, Bateman A, Enright AJ: miRBase: microRNA sequences, targets and gene nomenclature. Nucleic Acids Res 2006, 34:D I 40-D I 44.

18. UCSC Genome Browser [http://genome.ucsc.edu/]

19. Wong KK, deLeeuw RJ, Dosanjh NS, Kimm LR, Cheng Z, Horsman DE, MacAulay C, Ng RT, Brown CJ, Eichler EE, Lam WL: A comprehensive analysis of common copy-number variations in the human genome. Am J Hum Genet 2007, 80(I):9I-104.

20. Liva S, Hupe P, Neuvial P, Brito I, Viara E, La Rosa P, Barillot E: CAP. web: a bioinformatics CGH array Analysis Platform. Nucleic Acids Res 2006, 34(Web Server issue):W477-W48I.

2I. Conde L, Montaner D, Burquet-Castell J, Taragga J, Medina I, AlShahrour F, Dopazo J: ISACGH: a web-based environment for the analysis of Array CGH and gene expression which includes functional profiling. Nucleic Acids Res 2007, 35(Web Server issue):W8I-5.

22. Kim SY, Nam SW, Lee SH, Park WS, Yoo NJ, Lee JY, Chung YJ: ArrayCyGHt: a web application for analysis and visualization of array-CGH data. Bioinformatics 2005, 21 ( I 0):2554-2555.

Publish with Biomed Central and every scientist can read your work free of charge

"BioMed Central will be the most significant development for disseminating the results of biomedical research in our lifetime. "

Sir Paul Nurse, Cancer Research UK

Your research papers will be:

- available free of charge to the entire biomedical community

- peer reviewed and published immediately upon acceptance

- cited in PubMed and archived on PubMed Central

- yours - you keep the copyright

Submit your manuscript here:

http://www.biomedcentral.com/info/publishing_adv.asp 